\title{
Chemical and mineralogical characterization of the sediments from the Mira, Ílhavo and Ovar channels of Aveiro Lagoon (Portugal)
}

\section{Caracterización química y mineralógica de los sedimentos de los canales de Mira, Ílhavo y Ovar de la Laguna de Aveiro (Portugal)}

\author{
Fernando Rocha* \\ Eduardo Silva \\ Cristina Bernardes \\ Jesus Vidinha \\ Carla Patinha \\ Dep. Geociências \\ Univ. de Aveiro \\ 3810-193 Aveiro, Portugal \\ * frocha@geo.ua.pt
}

Recibido en junio de 2003; aceptado en mayo de 2004

\begin{abstract}
The paleoenvironmental reconstruction of Aveiro Lagoon is being carried out based on the sedimentological, paleoecological, geochemical and mineralogical characterization of sediment samples collected systematically in the lagoon. This paper presents results concerning the changes in the chemical and mineralogical composition of the fine fraction $(<63 \mu \mathrm{m})$ and the clay fraction $(<2 \mu \mathrm{m})$ in sediment samples from Aveiro Lagoon, mainly collected from the lower intertidal plain and subtidal deposits located along the Mira, Ílhavo and Ovar channels. A zonography of the lagoon was established based on the parameters analyzed. The results show that the surface and near-surface sediments of Aveiro Lagoon trap relatively large quantities of trace metals, such as $\mathrm{Cu}, \mathrm{Pb}, \mathrm{Co}, \mathrm{Ni}, \mathrm{Cd}, \mathrm{Zn}, \mathrm{Fe}, \mathrm{Mn}$ and $\mathrm{Cr}$, considered to be partially adsorbed, particularly on silty and clayey particles, and coprecipitated on Fe and Mn oxyhydroxide forms precipitated in the topmost layers of sediments. Series of statistically significant relationships were established between trace metal concentrations and clay content and semi-quantitatively determined clay mineral species in the sediments.
\end{abstract}

Key words: trace metals, recent sediments, Aveiro Lagoon.

\section{Resumen}

La reconstrucción paleoambiental de la Laguna de Aveiro se está realizando mediante la caracterización sedimentológica, paleoecológica, geoquímica y mineralógica de muestras de sedimento recogidas sistemáticamente en la misma. Este artículo presenta resultados relativos a las variaciones en la composición química y mineralógica de las fracciones fina (<63 $\mu \mathrm{m}) \mathrm{y}$ arcillosa $(<2 \mu \mathrm{m})$ de las muestras de sedimento de la Laguna de Aveiro, recogidas principalmente en las planicies intermareales bajas y depósitos submareales de los canales de Mira, Ílhavo y Ovar. Estos parámetros fueron utilizados para caracterizar los sedimentos de cada uno de los canales estudiados de la Laguna de Aveiro. Los sedimentos superficiales y subsuperficiales de esta laguna retienen cantidades relativamente elevadas de elementos vestigiales como $\mathrm{Cu}, \mathrm{Pb}$, Co, Ni, Cd, Zn, Fe, Mn y Cr, los cuales se consideran principalmente adsorbidos en partículas fangosas y arcillosas y en óxidos de Fe y Mn. Con base en los parámetros analizados se puede establecer una zonografía de la laguna. Se han encontrado relaciones entre las concentraciones de elementos vestigiales y el contenido de arcilla y las especies minerales de las arcillas en los sedimentos.

Palabras clave: elementos vestigiales, sedimentos actuales, Laguna de Aveiro.

\section{Introduction}

The paleoenvironmental reconstruction of Aveiro Lagoon is being carried out (Rocha and Gomes, 1991, 1992, 1994; Rocha et al., 1999, 2000) based on the sedimentological, paleoecological, geochemical and mineralogical characterization of sediment samples collected systematically in the lagoon (fig. 1).

\section{Introducción}

La reconstrucción paleoambiental de la Laguna de Aveiro se está realizando (Rocha y Gomes, 1991, 1992, 1994; Rocha et al., 1999, 2000) mediante la caracterización sedimentológica, paleoecológica, geoquímica y mineralógica de muestras de sedimento recolectadas sistemáticamente de la laguna (fig. 1). 


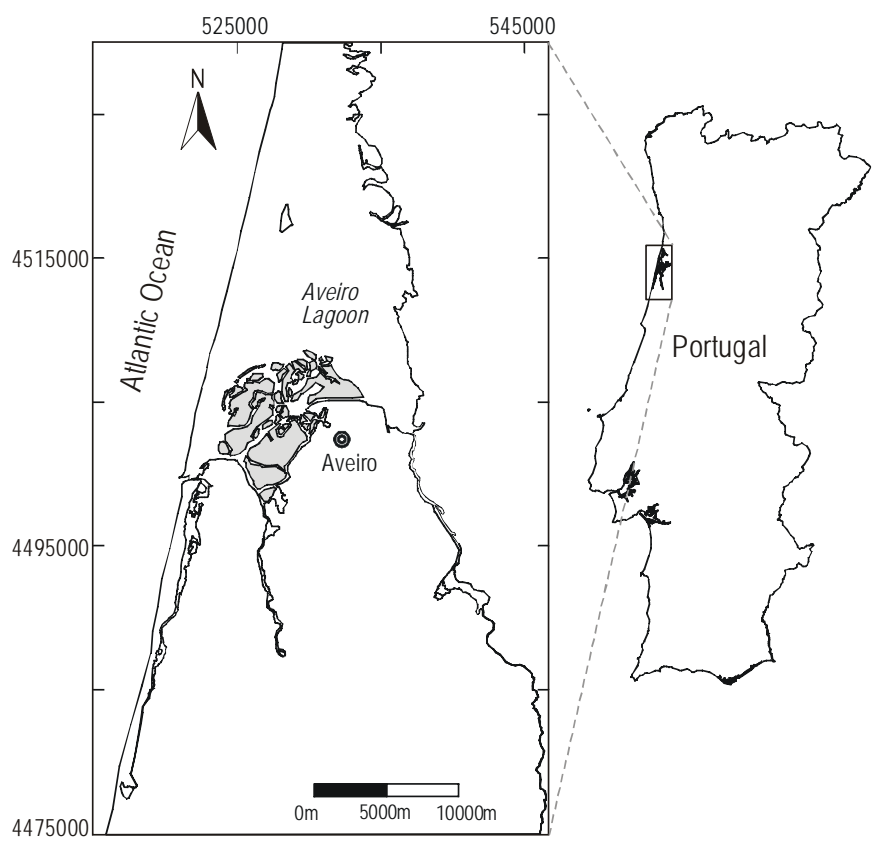

Figure 1. Study area location.

Figura 1. Localización del área de estudio.

The formation of the Aveiro coastal lagoon (the so-called Ría de Aveiro) initiated less than 1000 years ago when a sandy spit developed and proceeded southwards, isolating the Vouga River estuary from the Atlantic Ocean.

Several publications have generated a significant amount of data on the occurrence, distribution and dispersion of contaminants in Aveiro Lagoon over the last 25 years. In general, the main sources of pollution in the lagoon are linked to landbased activities: (1) location of large urban areas (with the associated problems of sewage discharge and final disposal of solid residues, land drainage, inappropriate land use, high levels of organic matter in water bodies, etc.); (2) location of large industrial parks (which can cause problems from contamination by heavy metals, synthetic chemical compounds, hydrocarbons, TCDD and/or TCDF and PCBs); and (3) large agriculture production areas (with associated fertilizer, agrochemical and pesticide loads).

One of the most important areas of the Portuguese chemical industry is located in the study area, more precisely in Estarreja. The most important industries produce ammonium sulphate, using sulphuric acid and ammonia, nitric acid and ammonium nitrate. Production of sodium and chlorate compounds from rock salt and several synthetic resins, mainly polyvinyl chloride (PVC), from monomer (VCM), is also well known (Batista et al., 2002). According to Costa and Jesus (1999), the chemical industry has discharged large quantities of solid waste, which was stockpiled directly on soil. This solid waste contains pyrite, ash, dust and sludge, containing $\mathrm{Hg}$ and calcium hydroxide. Until 1975, the liquid effluents produced by this industry were also discharged directly into several streams (Vala de S. Filipe, Vala da Breja, Vala do Canedo, Vala
La formación de la laguna costera de Aveiro (conocida como Ría de Aveiro) inició hace menos de 1000 años al desarrollarse un banco arenoso que se extendió hacia el sur y aisló el estuario del Río Vouga del Océano Atlántico.

Varias publicaciones han generado una cantidad significativa de datos sobre la ocurrencia, distribución y dispersión de contaminantes en la Laguna de Aveiro durante los pasados 25 años. En general, las fuentes principales de contaminación en la laguna están relacionadas con actividades terrestres: (1) la localización de grandes zonas urbanas (con los problemas asociados a desagües y eliminación de residuos sólidos, drenaje terrestre, manejo inadecuado de la tierra, altos niveles de materia orgánica en los cuerpos de agua, etc.); (2) la localización de parques industriales (lo cual conlleva contaminación por metales pesados, compuestos químicos sintéticos, hidrocarburos, TCDD y/o TCDF y PCBs); y (3) la localización de áreas de producción agrícola (con sus cargas asociadas de agroquímicos y de fertilizantes y pesticidas).

Una de las zonas principales de la industria química portuguesa se localiza en el área de estudio, específicamente en Estarreja. Las industrias más importantes producen sulfato de amonio, usando ácido sulfúrico y amoníaco, ácido nítrico y nitrato de amonio. También se producen sodio y compuestos clorados de sal de piedra y varias resinas sintéticas, principalmente cloruro de polivinilo (PVC) a partir de monómero (VCM) (Batista et al., 2002). Según Costa y Jesus (1999), la industria química ha vertido una gran cantidad de desechos sólidos que se han acumulado directamente en el suelo. Estos desechos sólidos contienen pirita, ceniza, polvo y lodo, con Hg e hidrato de calcio. Hasta 1975 los efluentes producidos por esta industria se vertían directamente en varios arroyos (Vala de S. Filipe, Vala da Breja, Vala do Canedo, Vala de Veiros y Esteiro de Estarreja). Se han realizado varios estudios (Ferreira da Silva, 1989; Barradas, 1992; Ferreira, 1993; Azevedo, 1999; Delgado et al., 2000) para medir los contenidos de elementos vestigiales en suelo y aguas superficiales, y para estimar las principales zonas de descarga en la Laguna de Aveiro.

También se localizan diversas zonas industriales (e.g., de pulpa y papel, metalmecánica y curtidurías) en Águeda (Ferreira da Silva, 1989) y Cacia, cuyos efluentes se descargan en los principales ríos (Águeda y Vouga) de la región.

Estas descargas, junto con los efluentes urbanos, son responsables de la contaminación de los sistemas estuarinos y fluviales de la región. Los sedimentos superficiales y subsuperficiales de la Laguna de Aveiro atrapan cantidades relativamente elevadas de elementos vestigiales (Delgado et al., 1994, 1996, 2000), tales como Cu, Pb, Co, Ni, Cd, Zn, Fe, $\mathrm{Mn}$ y $\mathrm{Cr}$, los cuales se considera que son parcialmente adsorbidos, especialmente en partículas arcillosas y limosas.

Se han publicado varios trabajos sobre la variabilidad de los parámetros texturales y sedimentológicos (Corrochano et al., 1997, 1999), así como sobre la composición mineralógica (Delgado et al., 1992; Rocha et al., 2000) de las fracciones fina $(<63 \mu \mathrm{m})$ y arcillosa $(<2 \mu \mathrm{m})$ de muestras de sedimento de la Laguna de Aveiro. 
de Veiros and Esteiro de Estarreja). Several surveys have been carried out (Ferreira da Silva, 1989; Barradas, 1992; Ferreira, 1993; Azevedo, 1999; Delgado et al., 2000) in order to measure the trace metal contents in soil and surface water and estimate the most important input areas into Aveiro Lagoon.

Several areas with diverse industries (e.g., tanneries, metalmechanic and pulp and paper industries) also exist in Águeda (Ferreira da Silva, 1989) and Cacia, whose effluents are discharged into the main rivers (Águeda and Vouga) of the region.

These discharges, together with the disposal of urban effluents, are responsible for the pollution in the estuarine and riverine systems of the area. The surface and near-surface sediments of Aveiro Lagoon trap relatively large quantities of trace metals (Delgado et al., 1994, 1996, 2000), such as $\mathrm{Cu}, \mathrm{Pb}, \mathrm{Co}$, $\mathrm{Ni}, \mathrm{Cd}, \mathrm{Zn}, \mathrm{Fe}, \mathrm{Mn}$ and $\mathrm{Cr}$, considered to be partially adsorbed, particularly on silty and clayey particles.

Several studies carried out in Aveiro Lagoon have reported on the variability of the textural and sedimentological parameters (Corrochano et al., 1997, 1999) and on the mineralogical composition (Delgado et al., 1992; Rocha et al., 2000) of the fine $(<63 \mu \mathrm{m})$ and clay $(<2 \mu \mathrm{m})$ fractions of sediment samples from the lagoon.

The objectives of this study were: (1) the overall characterization of Aveiro Lagoon to assess the environmental degradation; (2) to evaluate the changes in the chemical and mineralogical composition of the fine fraction $(<63 \mu \mathrm{m})$ and the clay fraction $(<2 \mu \mathrm{m})$ of sediment samples from the lagoon, collected mainly from the lower intertidal plain and subtidal deposits located along the Mira, Ílhavo and Ovar channels; (3) to investigate the zonography of the lagoon based on the parameters analyzed; and (4) to establish the relationships between the high trace metal concentrations and the clay content and clay mineral species in the sediments.

\section{Materials and methods}

Fifty underwater sediment samples weighing around $1 \mathrm{~kg}$ were collected using a dredge in the Aveiro Lagoon channels.

Mineralogical studies were based on X-ray diffraction (XRD) determinations, carried out on the fine $(<63 \mu \mathrm{m})$ and clay $(<2 \mu \mathrm{m})$ grain size fractions of the sediments, using a Phillips PW 3040/60 diffractometer. The clay fraction was separated by sedimentation, according to Stokes' law. Oriented specimens for subsequent $\mathrm{X}$-ray diffraction analysis were then analyzed after drying, glycolation and heating to $300^{\circ} \mathrm{C}$ and $500^{\circ} \mathrm{C}$. All samples were analyzed in the range from $2^{\circ}$ to $40^{\circ}$ $2 \theta$, at $1^{\circ} 2 \theta / \mathrm{min}$, with $\mathrm{Cu}-\mathrm{Ka}$ radiation. The XRD reflections were evaluated with the Phillips X'Pert 1.2 and Profit softwares. For the semi-quantitative determination of clay and non-clay minerals, the relative content of each identified mineral was estimated on the basis of its characteristic peak area corrected by the corresponding reflective power recommended by Schultz (1964), Barahona (1974), Thorez (1976), Mellinger (1979) and Pevear and Mumpton (1989).
Los objetivos del presente estudio fueron: (1) realizar una caracterización global de la Laguna de Aveiro para determinar su degradación ambiental; (2) evaluar los cambios en la composición química y mineralógica de las fracciones fina $(<63$ $\mu \mathrm{m})$ y arcillosa $(<2 \mu \mathrm{m})$ de muestras de sedimento de la laguna, recolectadas principalmente de las planicies intermareales y los depósitos submareales localizados en los canales de Mira, Ílhavo y Ovar; (3) investigar la zonografía de la laguna con base en los parámetros analizados; y (4) establecer las relaciones entre las elevadas concentraciones de elementos vestigiales y el contenido de arcilla y especies de minerales arcillosos en los sedimentos.

\section{Materiales y métodos}

Se recolectaron 50 muestras de sedimento subacuático, pesando alrededor de $1 \mathrm{~kg}$, con una draga en los canales de la Laguna de Aveiro.

Los estudios mineralógicos se basaron en determinaciones mediante difracción de rayos X (XRD) de las fracciones fina $(<63 \mu \mathrm{m})$ y arcillosa $(<2 \mu \mathrm{m})$ de los sedimentos, utilizando un difractómetro Phillips PW 3040/60. Se separó la fracción arcillosa por sedimentación, según la ley de Stokes. Antes de su análisis por XRD, las muestras orientadas fueron puestas a secar, glicoladas y calentadas a $300^{\circ} \mathrm{C}$ y $500^{\circ} \mathrm{C}$. Todas las muestras se analizaron en el intervalo de $2^{\circ}$ a $40^{\circ} 2 \theta$, a $1^{\circ}$ 20/min, con radiación Cu-Ka. Las reflexiones por XRD fueron evaluadas con los programas Phillips X'Pert 1.2 y Profit. Para la determinación semicuantitativa de arcilla y minerales no arcillosos, se estimó el contenido relativo de cada mineral identificado con base en su área pico característica, corregida mediante el correspondiente poder reflectivo recomendado por Schultz (1964), Barahona (1974), Thorez (1976), Mellinger (1979) y Pevear y Mumpton (1989).

Las alícuotas de sedimento seco ( $1 \mathrm{~g}$ ) fueron digeridas en una mezcla de ácidos concentrados de $3 \mathrm{~mL}$ de $\mathrm{HCl}, 2 \mathrm{~mL}$ de $\mathrm{HNO}_{3}$ y $1 \mathrm{~mL}$ de $\mathrm{HF}$, y finalmente se disolvieron en $\mathrm{HNO}_{3} 4 \mathrm{~N}$. Las concentraciones de $\mathrm{Fe}, \mathrm{Cu}, \mathrm{Pb}, \mathrm{Zn}, \mathrm{Mn}, \mathrm{Cd}$, $\mathrm{Co}$, Ni y $\mathrm{Cr}$ en la solución de ácidos se determinaron por espectrometría de absorción atómica (Lecomte y Sondag, 1980).

Para controlar el proceso de análisis y determinar posibles errores en los lotes, se incluyeron muestras de un lote junto con las de otros lotes. La precisión de los resultados analíticos se estimó mediante análisis de réplicas (Garrett, 1973). La precisión analítica, definida como el porcentaje de desviación estándar con relación a un nivel de confianza de $95 \%$, fue de $6.8 \%$ para $\mathrm{Cu}, 3.7 \%$ para $\mathrm{Pb}, 5.5 \%$ para $\mathrm{Zn}, 2.1 \%$ para $\mathrm{Fe}$, $7.2 \%$ para $\mathrm{Mn}, 7.5 \%$ para $\mathrm{Co}, 7.1 \%$ para $\mathrm{Ni}, 6.8 \%$ para $\mathrm{Cr}$ y $13 \%$ para Cd.

Se realizó un análisis multivariado (análisis de componentes principales) de los datos químicos. Según Imbrie y Van Andel (1964), Jöreskog et al. (1976), Davis (1986) y Reyment y Jöreskob (1993), el uso de estos métodos estadísticos permite una buena caracterización, mediante la reducción de la complejidad del modelo y la clasificación de las variables y muestras en grupos naturales (Mezzadri y Saccani, 1989). 
The dried sediment aliquots ( $1 \mathrm{~g})$ were digested in a mixture of $3 \mathrm{~mL} \mathrm{HCl}, 2 \mathrm{~mL} \mathrm{HNO}_{3}$ and $1 \mathrm{~mL}$ HF concentrated acids, and finally dissolved in $4 \mathrm{~N}^{-N_{3}}$. The concentrations of $\mathrm{Fe}, \mathrm{Cu}, \mathrm{Pb}, \mathrm{Zn}, \mathrm{Mn}, \mathrm{Cd}, \mathrm{Co}, \mathrm{Ni}$ and $\mathrm{Cr}$ in the acid solution were determined by atomic absorption spectrometry (Lecomte and Sondag, 1980).

To control the analytical process and check for possible batch errors, samples from one batch were included in other batches. The precision of the analytical results was estimated by replicate analysis (Garrett, 1973). Analytical precision, defined as the percent relative standard deviation at the $95 \%$ confidence level, is $6.8 \%$ for $\mathrm{Cu}, 3.7 \%$ for $\mathrm{Pb}, 5.5 \%$ for $\mathrm{Zn}$, $2.1 \%$ for $\mathrm{Fe}, 7.2 \%$ for $\mathrm{Mn}, 7.5 \%$ for $\mathrm{Co}, 7.1 \%$ for $\mathrm{Ni}, 6.8 \%$ for $\mathrm{Cr}$ and $13 \%$ for $\mathrm{Cd}$.

Multivariate analysis (principal components analysis) of the chemical data was carried out. The use of these statistical methods, as outlined by Imbrie and Van Andel (1964), Jöreskog et al. (1976), Davis (1986) and Reyment and Jöreskob (1993), allows a convenient characterization of the data, through the reduction of the complexity of the model and classification of the variables and samples into natural groups (Mezzadri and Saccani, 1989).

\section{Results and discussion}

The principal minerals of the fine fraction $(<63 \mu \mathrm{m})$ of the sediments were phyllosilicates (Phyl), quartz (Qz), plagioclase (P) and K-feldspar (FK). Several accessory minerals were identified: anhydrite (An), opal C/CT (Op), calcite (Ca), dolomite (Do), siderite (Sy), pyrite (Py) and zeolites (Ze) (fig. 2). The finer versus coarser detrital ratio (Phyl/Qz $+\mathrm{Fk}+\mathrm{P}$ ) gives some indication about the hydrodynamic behaviour of the channels in Aveiro Lagoon. The higher values of this ratio correspond to low hydrodynamic levels that were favourable to higher rates of sediment deposition (fig. 3).

Regarding the clay fraction $(<2 \mu \mathrm{m})$, illite (Il) was the predominant mineral, accompanied by kaolinite (K), chlorite (C), vermiculite (V) and smectite (Sm) (fig. 4). These clay minerals show, in general, low/medium crystallinity with no significant variation. Illite shows a slightly higher crystallinity, with a discrete lateral heterogeneity.

In the analysis of the chemical data (table 1), the nine variables $(\mathrm{Cu}, \mathrm{Pb}, \mathrm{Co}, \mathrm{Ni}, \mathrm{Cd}, \mathrm{Zn}, \mathrm{Fe}, \mathrm{Mn}$ and $\mathrm{Cr})$ were logarithmically transformed. This transformation allows a substantial decrease in the coefficient of variation, preventing subsequent estimation problems.

The nine principal components extracted from the correlation matrix accounted for $38.1 \%, 15.7 \%, 12.7 \%, 11.5 \%$, $10.19 \%, 8.38 \%, 2.18 \%, 0.88 \%$ and $0.34 \%$ of the total variance. So, the first three factors represent $66.5 \%$ of the total variance. The fourth factor (11.5\%) hardly explains more variance than any of the original variables alone $(1 / 9=11.1 \%)$.

The multivariate factorial analysis (principal components analysis) of the chemical data allows the following considerations (tables 1, 2):

\section{Resultados y discusión}

Los principales minerales de la fracción fina $(<63 \mu \mathrm{m})$ de los sedimentos fueron filosilicatos (Phyl), cuarzo (Qz), plagioclasa (P) y feldespato (FK). Se identificaron varios minerales asociados: anhidrita (An), ópalo C/CT (Op), calcita (Ca), dolomita (Do), siderita (Sy), pirita (Py) y ceolitas (Ze) (fig. 2). La razón detrito más fino/grueso (Phyl/Qz + Fk + P) indica el comportamiento hidrodinámico de los canales de la Laguna de Aveiro. Los valores más altos de esta razón corresponden a los niveles hidrodinámicos bajos que favorecieron las tasas más altas de depósito de sedimento (fig. 3).

Con respecto a la fracción arcillosa $(<2 \mu \mathrm{m})$, el mineral predominante fue la ilita (Il), junto con la caolinita (K), clorita (C), vermiculita (V) y esmectita (Sm) (fig. 4). En general, estos minerales arcillosos muestran de baja a mediana cristalinidad sin variaciones significativas. La ilita muestra una cristalinidad ligeramente mayor, con heterogeneidad lateral discreta.

En el análisis de los datos químicos (tabla 1), las nueve variables $(\mathrm{Cu}, \mathrm{Pb}, \mathrm{Co}, \mathrm{Ni}, \mathrm{Cd}, \mathrm{Zn}, \mathrm{Fe}, \mathrm{Mn}$ y $\mathrm{Cr}$ ) fueron transformadas logarítmicamente. Esta transformación permite una reducción substancial del coeficiente de variación, lo cual evita problemas de estimación posteriores.

Los nueve componentes principales extraídos de la matriz de correlación explicaron 38.1\%, 15.7\%, 12.7\%, 11.5\%, $10.19 \%, 8.38 \%, 2.18 \%, 0.88 \%$ y $0.34 \%$ de la varianza total. Así, los primeros tres factores representan $66.5 \%$ de la varianza total. El cuarto factor (11.5\%) apenas explica una varianza ligeramente mayor a la explicada por cualquiera de las variables originales solas $(1 / 9=11.1 \%)$.

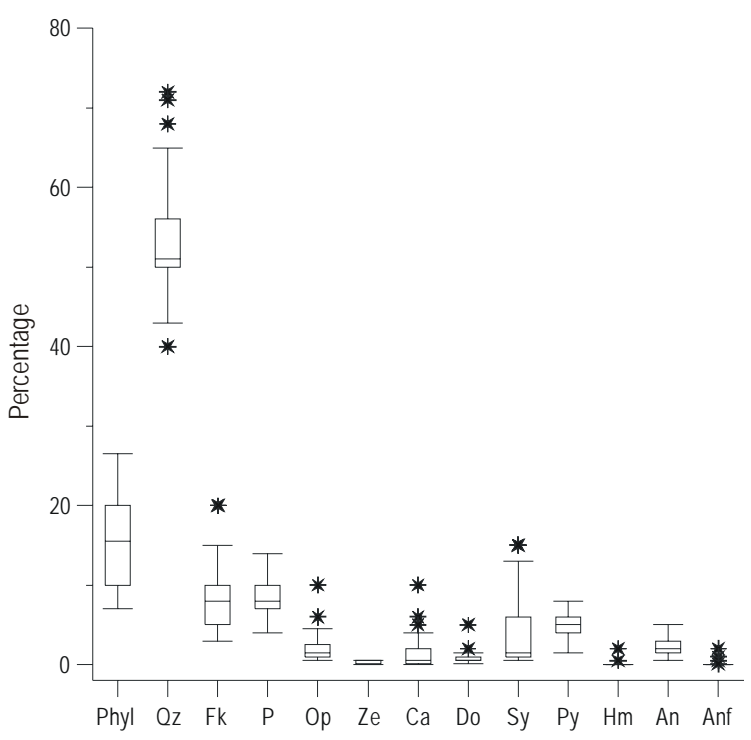

Figure 2. Box-plot of the minerals identified in the fine fraction of the sediments of the Aveiro Lagoon channels: median, first and third quartile, minimum and maximum and outliers.

Figura 2. Gráfica de los minerales identificados en la fracción fina de los sedimentos de los canales de la Laguna de Aveiro: mediana, primer y tercer cuartiles, mínimo y máximo, y valores atípicos. 


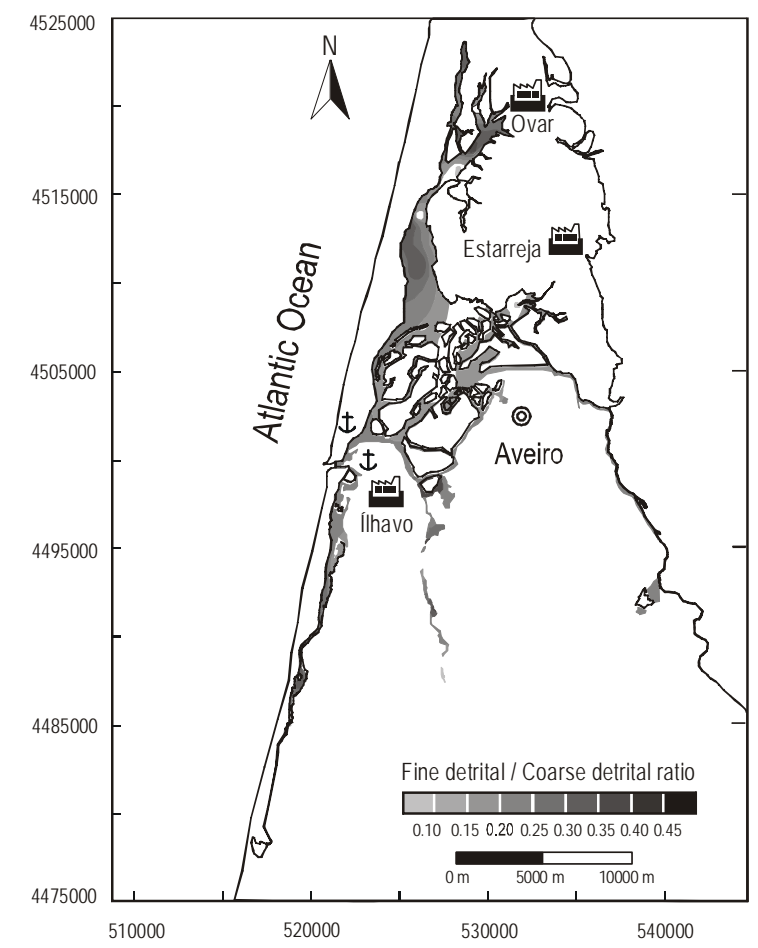

Figure 3. Fine detritus versus coarse detritus ratio mapping. Fine detritus corresponds to phyllosilicates and coarse detritus to quartz and feldspars. Figura 3. Cartografía de la razón de detrito fino vs detrito grueso. El detrito fino corresponde a los filosilicatos y el grueso a cuarzo y feldespatos.

- The elements $\mathrm{Cu}, \mathrm{Co}, \mathrm{Cd}$ and $\mathrm{Zn}$ have a strong negative loading in Factor I (table 2). The corresponding mapping of the factor scores (fig. 5a) shows the influence of the industrial parks located close to Ovar and Estarreja (north of Aveiro).

- Factor II explains the Mn variable. Although the distribution pattern of Mn in the surface sediments of coastal areas is commonly defined by post-mobilization processes and precipitation of $\mathrm{Mn}$ and $\mathrm{Fe}$ oxides, the mapping of these factor scores (fig. 5b) evidences co-precipitation of the Mn and Fe oxides and the importance of the adsorption of trace metal from the liquid effluents of the Ovar and Estarreja chemical parks. These adsorption processes occur preferentially in more confined (therefore less hydrodynamic) channels where the bottom sediments are mainly silty/ clayey muds richer in clay minerals displaying higher specific surface areas and cation exchange capacities, such as smectite, illite-smectite, vermiculite, illite-vermiculite interstratifications and vermiculite-Al hydroxide interlayers (Delgado et al., 1992, 1996; Gomes and Delgado, 1993; Rocha et al., 2000).

- Factor III explains the association between the $\mathrm{Ni}$ and $\mathrm{Cr}$ variables. The peculiar cartographic distribution of the

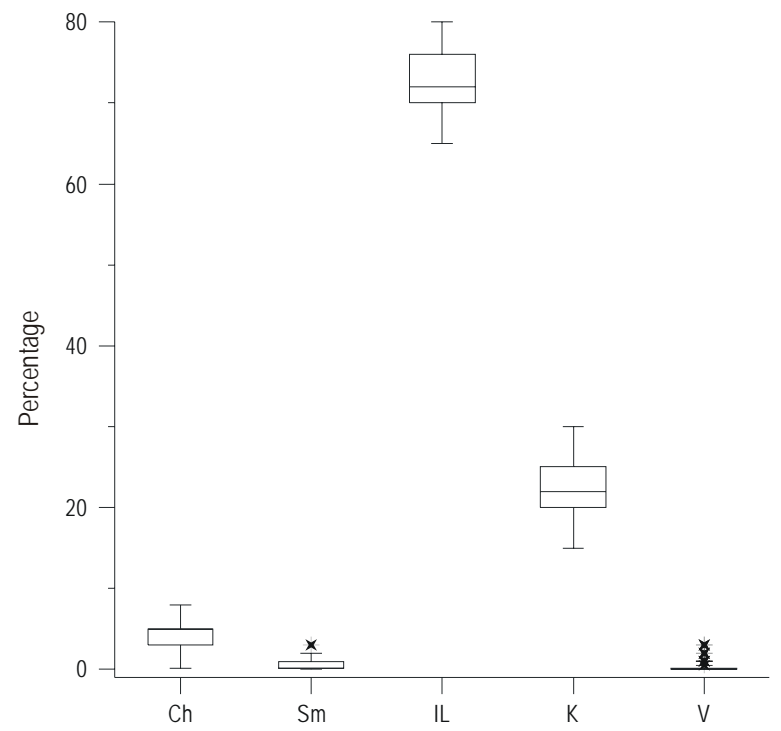

Figure 4. Box-plot of minerals identified in the clay fraction of the sediments of the Aveiro Lagoon channels: median, first and third quartile, minimum and maximum and outliers.

Figura 4. Gráfica de los minerales identificados en la fracción arcillosa de los sedimentos de los canales de la laguna: mediana, primer y tercer cuartiles, mínimo y máximo y valores atípicos.

El análisis multivariado (análisis de componentes principales) de los datos químicos permite las siguientes consideraciones (tablas 1, 2):

- Los elementos $\mathrm{Cu}, \mathrm{Co}, \mathrm{Cd}$ y $\mathrm{Zn}$ tienen una fuerte carga negativa en el Factor I (tabla 2). La cartografía correspondiente a la puntuación factorial (fig. 5a) muestra la influencia de los parques industriales localizados cerca de Ovar y Estarreja (al norte de Aveiro).

- El Factor II explica la variable Mn. Aunque el patrón de distribución de $\mathrm{Mn}$ en los sedimentos superficiales de zonas costeras es comúnmente definido por procesos posteriores a la movilización y por la precipitación de óxidos de Mn y Fe, la cartografía de estos valores factoriales (fig. 5b) indica coprecipitación de los óxidos de Mn y Fe, así como la importancia de la adsorción de elementos vestigiales de los efluentes de los parques químicos de Ovar y Estarreja. Estos procesos de adsorción preferentemente suceden en canales más confinados (y por tanto menos dinámicos), donde los sedimentos del fondo son principalmente lodos arcillosos/limosos ricos en minerales de arcilla que exhiben mayores áreas superficiales específicas y capacidad de intercambio catiónico, como en interestratificaciones de esmectita, ilita-esmectita, vermiculita e ilita-vermiculita, e intercapas de vermiculita e hidróxido de $\mathrm{Al}$ (Delgado et al., 1992, 1996; Gomes y Delgado, 1993; Rocha et al., 2000).

- El Factor III explica la asociación entre las variables Ni y Cr. La distribución cartográfica particular de los valores factoriales (fig. 6a) sugiere ya sea un control litológico/ 
Table 1. Trace metal concentrations (ppm) determined in the sediments collected from the Mira, Ílhavo and Ovar channels of Aveiro Lagoon. Tabla 1. Concentraciones de elementos vestigiales (ppm) determinados en sedimentos recolectados en los canales de Mira, Ílhavo y Ovar de la Laguna de Aveiro.

\begin{tabular}{|c|c|c|c|c|c|c|c|c|c|}
\hline Sample & $\mathrm{Cu}$ & $\mathrm{Pb}$ & Co & $\mathrm{Ni}$ & $\mathrm{Cd}$ & $\mathrm{Zn}$ & $\mathrm{Fe}$ & $\mathrm{Mn}$ & $\mathrm{Cr}$ \\
\hline 1 & 298 & 56 & 49 & 42 & 10.0 & 3961 & 26217 & 223 & 44 \\
\hline 2 & 18 & 42 & 32 & 38 & 0.0 & 97 & 26520 & 408 & 42 \\
\hline 3 & 52 & 52 & 41 & 40 & 1.5 & 883 & 25614 & 236 & 53 \\
\hline 4 & 25 & 51 & 37 & 32 & 0.0 & 213 & 20379 & 622 & 39 \\
\hline 5 & 31 & 53 & 31 & 34 & 0.0 & 157 & 12801 & 152 & 39 \\
\hline 6 & 23 & 45 & 28 & 32 & 0.0 & 234 & 24299 & 187 & 40 \\
\hline 7 & 14 & 34 & 37 & 32 & 0.0 & 50 & 32168 & 131 & 45 \\
\hline 8 & 34 & 53 & 31 & 32 & 0.0 & 183 & 22384 & 175 & 44 \\
\hline 9 & 33 & 53 & 34 & 39 & 0.0 & 263 & 20612 & 236 & 46 \\
\hline 10 & 19 & 40 & 35 & 39 & 0.0 & 94 & 27209 & 257 & 48 \\
\hline 11 & 92 & 48 & 32 & 40 & 0.4 & 485 & 23357 & 211 & 44 \\
\hline 12 & 13 & 37 & 24 & 26 & 0.0 & 155 & 15674 & 126 & 34 \\
\hline 13 & 42 & 70 & 36 & 66 & 0.0 & 243 & 18136 & 129 & 38 \\
\hline 14 & 30 & 47 & 33 & 34 & 0.0 & 115 & 27315 & 176 & 41 \\
\hline 15 & 26 & 47 & 30 & 34 & 0.0 & 108 & 26672 & 151 & 40 \\
\hline 16 & 24 & 45 & 34 & 34 & 0.0 & 114 & 27136 & 142 & 37 \\
\hline 17 & 13 & 37 & 28 & 24 & 0.0 & 54 & 20283 & 141 & 26 \\
\hline 18 & 22 & 41 & 32 & 31 & 0.0 & 92 & 23740 & 142 & 33 \\
\hline 19 & 28 & 47 & 32 & 33 & 0.0 & 123 & 25923 & 154 & 36 \\
\hline 20 & 30 & 42 & 30 & 32 & 0.0 & 134 & 36301 & 341 & 33 \\
\hline 21 & 14 & 44 & 30 & 34 & 0.0 & 91 & 23268 & 246 & 39 \\
\hline 22 & 21 & 46 & 32 & 38 & 0.0 & 183 & 26944 & 181 & 49 \\
\hline 23 & 22 & 49 & 30 & 36 & 0.0 & 207 & 21647 & 171 & 42 \\
\hline 24 & 28 & 46 & 33 & 39 & 0.0 & 513 & 29535 & 221 & 40 \\
\hline 25 & 25 & 55 & 35 & 44 & 0.0 & 132 & 31875 & 263 & 53 \\
\hline 26 & 29 & 46 & 36 & 39 & 0.0 & 567 & 31304 & 256 & 36 \\
\hline 27 & 23 & 51 & 33 & 36 & 0.0 & 220 & 22953 & 175 & 39 \\
\hline 28 & 16 & 40 & 31 & 31 & 0.0 & 145 & 22443 & 191 & 37 \\
\hline 29 & 21 & 45 & 36 & 35 & 0.0 & 228 & 27652 & 245 & 35 \\
\hline 30 & 32 & 119 & 41 & 40 & 0.0 & 178 & 30680 & 246 & 34 \\
\hline 31 & 63 & 58 & 47 & 46 & 2.4 & 2015 & 29610 & 313 & 53 \\
\hline 32 & 20 & 46 & 42 & 46 & 0.0 & 142 & 22704 & 341 & 34 \\
\hline 33 & 29 & 59 & 33 & 36 & 0.0 & 191 & 21768 & 157 & 38 \\
\hline 34 & 22 & 44 & 32 & 34 & 0.0 & 338 & 15578 & 159 & 42 \\
\hline 35 & 89 & 72 & 41 & 48 & 0.0 & 375 & 21234 & 176 & 41 \\
\hline 36 & 74 & 68 & 36 & 41 & 0.0 & 214 & 32283 & 260 & 48 \\
\hline 37 & 174 & 91 & 60 & 40 & 0.0 & 656 & 24375 & 2607 & 39 \\
\hline 38 & 85 & 197 & 34 & 45 & 0.0 & 347 & 21168 & 273 & 67 \\
\hline 39 & 30 & 53 & 32 & 36 & 0.3 & 188 & 27185 & 221 & 42 \\
\hline 40 & 54 & 43 & 33 & 34 & 0.0 & 218 & 44069 & 486 & 32 \\
\hline 41 & 28 & 61 & 34 & 39 & 0.0 & 134 & 27917 & 188 & 64 \\
\hline 42 & 17 & 1091 & 35 & 40 & 0.0 & 125 & 23973 & 219 & 35 \\
\hline 43 & 12 & 38 & 31 & 32 & 0.0 & 102 & 21193 & 146 & 39 \\
\hline 44 & 42 & 51 & 41 & 44 & 1.0 & 701 & 28712 & 227 & 36 \\
\hline 45 & 21 & 43 & 41 & 48 & 0.0 & 119 & 33284 & 355 & 40 \\
\hline 46 & 36 & 59 & 35 & 43 & 0.4 & 273 & 23091 & 150 & 35 \\
\hline 47 & 34 & 46 & 38 & 40 & 0.0 & 560 & 24725 & 190 & 33 \\
\hline 48 & 28 & 47 & 41 & 35 & 0.0 & 87 & 30193 & 218 & 23 \\
\hline 49 & 32 & 42 & 35 & 37 & 0.7 & 565 & 21018 & 209 & 20 \\
\hline 50 & 45 & 65 & 34 & 43 & 0.6 & 346 & 24794 & 159 & 19 \\
\hline
\end{tabular}


Table 2. Factor loadings (unrotated). Marking loadings are $>0.50$. Tabla 2. Cargas factoriales (sin rotar). Las cargas son $>0.50$.

\begin{tabular}{lcccc}
\hline & Factor I & Factor II & Factor III & Factor IV \\
\hline $\mathrm{Cu}$ & -0.927 & - & - & - \\
$\mathrm{Pb}$ & - & - & - & -0.620 \\
$\mathrm{Co}$ & -0.802 & - & - & - \\
$\mathrm{Ni}$ & - & - & -0.598 & - \\
$\mathrm{Cd}$ & -0.832 & -0.511 & - & - \\
$\mathrm{Zn}$ & -0.893 & - & - & - \\
$\mathrm{Fe}$ & - & - & - & 0.627 \\
$\mathrm{Mn}$ & - & 0.738 & - & - \\
$\mathrm{Cr}$ & - & - & -0.619 & - \\
\hline Explained & 3.432 & 1.411 & 1.140 & 1.038 \\
variance & & & & 11.5 \\
$\%$ total & 38.1 & 15.7 & 12.7 & \\
variance & & & & \\
\hline
\end{tabular}

factor scores (fig. 6a) suggests either a lithological/ mineralogical control (vermiculite and smectite rich muds) or some influence of different chemical parks surrounding the lagoon (Ovar, Estarreja and Ílhavo), pointing to the influence of the naval activities (port and shipyards) linked with Aveiro harbour. mineralógico (lodos ricos en vermiculita y esmectita) o alguna influencia de diferentes parques químicos alrededor de la laguna (Ovar, Estarreja e Ílhavo), señalando la influencia de las actividades navales (puerto y astilleros) vinculadas con Aveiro.

- El Factor IV explica la variable Fe de forma opuesta a la variable $\mathrm{Pb}$. Los valores positivos de este factor muestran la asociación entre Fe y las actividades del puerto, mientras que la cartografía de los valores negativos indica la asociación de $\mathrm{Pb}$ con el parque industrial de Estarreja (fig. 6b).

La cartografía de los diferentes valores factoriales confirma, en general, el modelo de dispersión desarrollado por Delgado et al. (2000), quienes señalan a la región de Ovar como la principal fuente de $\mathrm{Cu}$ y $\mathrm{Zn}$. La distribución de los valores del Factor I confirma esta conclusión e indica un confinamiento de su dispersión, pero excluye la región de Ílhavo como la segunda fuente principal de $\mathrm{Zn}$, como fue sugerido por Delgado et al. (2000) (fig. 7); sin embargo, se ratificó que la distribución de $\mathrm{Cr}$ y Ni (Factor III) concuerda con las conclusiones de estos autores, mientras que $\mathrm{Pb}$ (Factor IV) muestra un confinamiento y, simultáneamente, un cambio de foco hacia el oeste (fig. 8).

La comparación del comportamiento de la razón de minerales detríticos finos vs gruesos (fig. 3) con la puntuación
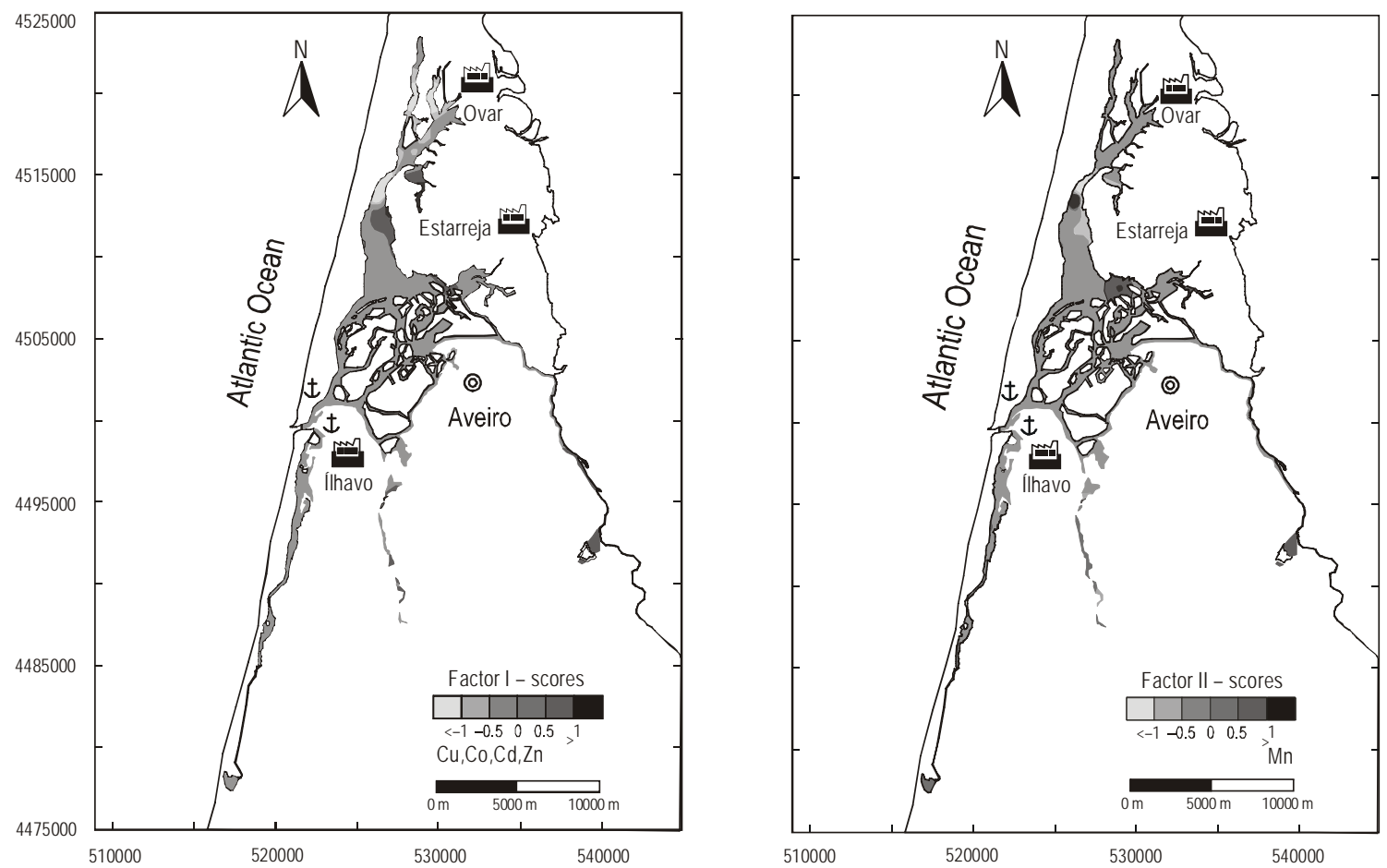

Figure 5. Mapping of the Factor I (a) and Factor II (b) scores. Significant scores are lesser or greater than -0.5 and 0.5 , respectively. Figura 5. Cartografía de la puntuación del Factor I (a) y Factor II (b). Los valores significativos son menores o mayores que -0.5 y 0.5, respectivamente. 
a

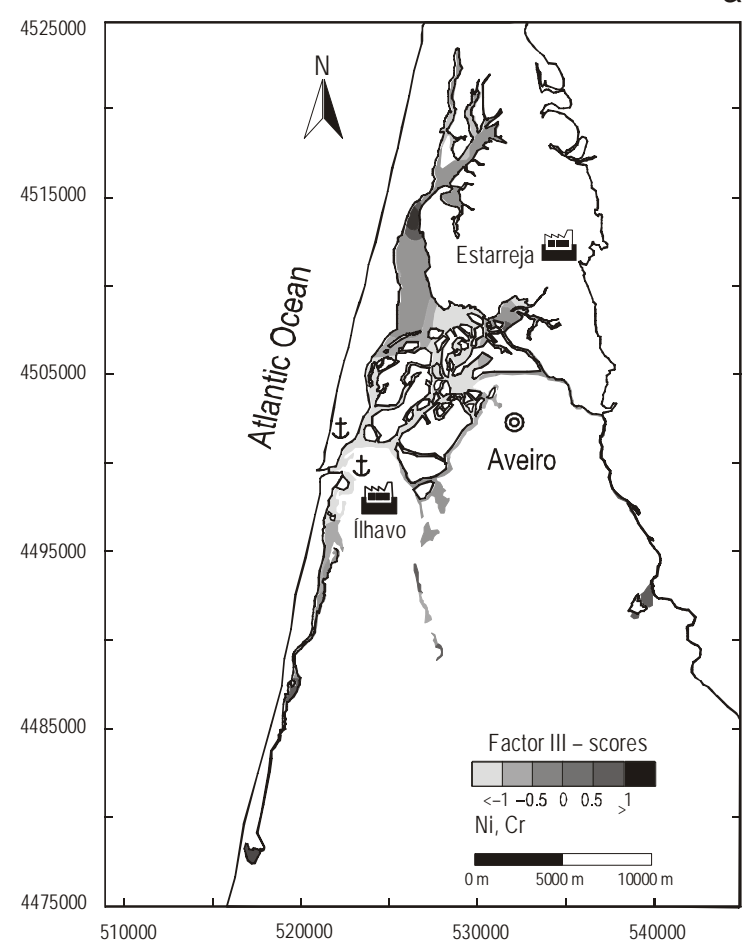

b

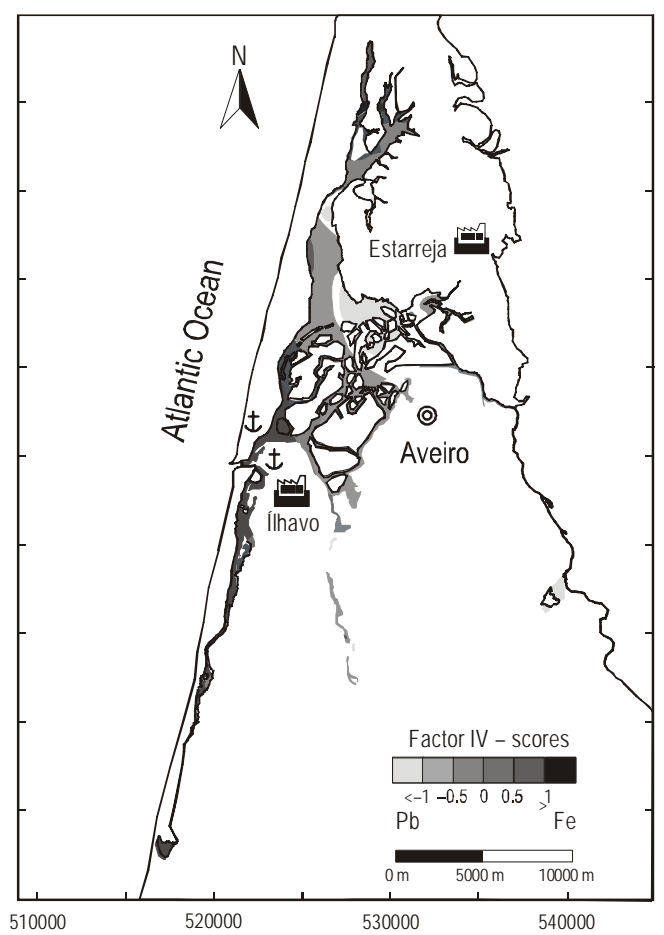

Figure 6. Mapping of the Factor III (a) and Factor IV (b) scores. Significant scores are lesser or greater than -0.5 and 0.5 , respectively. Figura 6. Cartografía de la puntuación del Factor III (a) y Factor IV (b). Los valores significativos son menores o mayores que -0.5 y 0.5 , respectivamente.

- Factor IV explains the Fe variable in opposition to the $\mathrm{Pb}$ variable. The positive scores of this factor show the association between $\mathrm{Fe}$ and harbour activities, while the mapping of negative scores points to some association of $\mathrm{Pb}$ with the Estarreja industrial park (fig. 6b).

The mapping of the different factor score values confirms, in general, the dispersion model developed by Delgado et al. (2000). These authors point to the Ovar region as the main source of $\mathrm{Cu}$ and $\mathrm{Zn}$. The distribution of the Factor I scores confirms this conclusion and indicates a confinement of their dispersion, but excludes the Ílhavo region as the second main source of Zn, as reported by Delgado et al. (2000) (fig. 7); however, we verified that the distribution of $\mathrm{Cr}$ and $\mathrm{Ni}$ (Factor III) is in accordance with the conclusions of Delgado et al. (2000), whereas Pb (Factor IV) reveals a confinement and, simultaneously, a focus shift westwards (fig. 8).

Comparing the behaviour of the fine versus coarse detrital mineral ratio (fig. 3) with the factors scores (fig. 5), the occurrence of some overlapping between the higher values of this ratio and the significant factor score areas is clear, suggesting a "hydraulic" control over the chemical dispersion of the elements in the lagoon channels. Dispersion of fine particles, which have a larger specific area, may be an important vehicle to transport anthropogenic metals from one site to another. factorial (fig. 5), muestra claramente un traslapo entre los valores más altos de esta razón y las áreas de valores factoriales significativos. Esto sugiere un control "hidráulico" sobre la dispersión química de los elementos en los canales de la laguna. La dispersión de partículas finas, con un área específica mayor, puede ser un vehículo importante para transportar metales antropogénicos de un sitio a otro.

En cuanto a la relación entre las concentraciones de elementos vestigiales y la distribución de minerales arcillosos, se encontró que los contenidos de elementos vestigiales sí son mayores en las zonas donde los sedimentos son más ricos en minerales arcillosos, con mayores áreas superficiales específicas y capacidades de intercambio catiónico, en relación con la existencia de interestratificaciones de esmectita, ilitaesmectita, vermiculita e ilita-vermiculita, así como intercapas de vermiculita e hidróxido de Al (Delgado et al., 1992, 1996; Gomes y Delgado, 1993; Rocha et al., 2000).

Con base en la cartografía de los contenidos de $\mathrm{Cu}, \mathrm{Ni}, \mathrm{Pb}$, $\mathrm{Zn}$ y $\mathrm{Cr}$ en los sedimentos de los canales de la Laguna de Aveiro y su comparación con los obtenidos en la década de los años ochenta por Delgado et al. (2000) (figs. 7, 8), se puede concluir que $\mathrm{Cu}$ y $\mathrm{Zn}$ muestran una tendencia a dispersarse de sus fuentes en Ovar y Estarreja y, al mismo tiempo, altos contenidos de éstos están más confinados en el sector norte (cerca de Ovar). El Ni muestra una dispersión, principalmente de su fuente en Estarreja y de la cuenca del Bajo Vouga. El Pb exhibe 

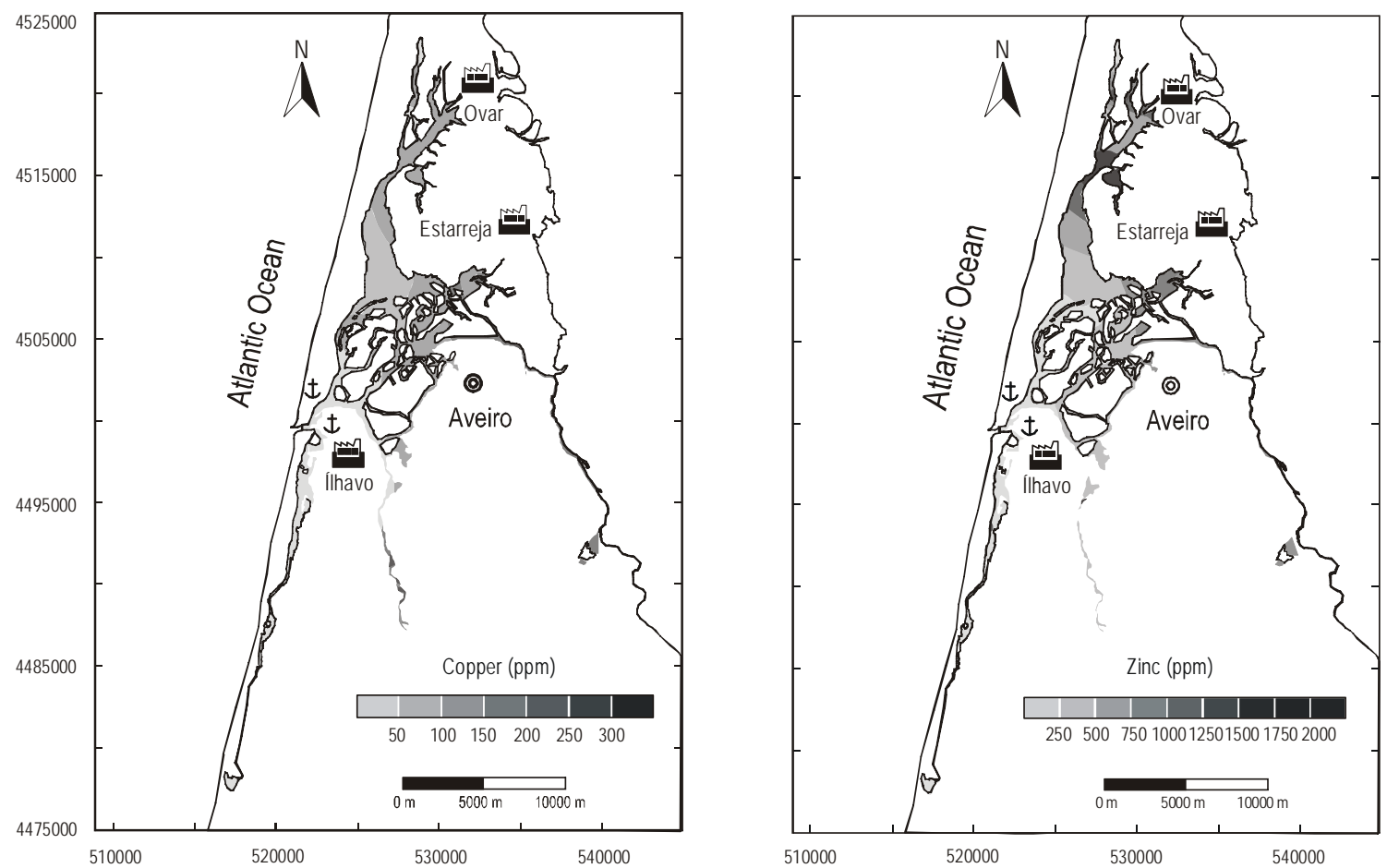

Figure 7. Dispersion maps of $\mathrm{Cu}$ and $\mathrm{Zn}$; data from Delgado et al. (2000).

Figura 7. Mapas de dispersión de Cu y Zn; datos de Delgado et al. (2000).
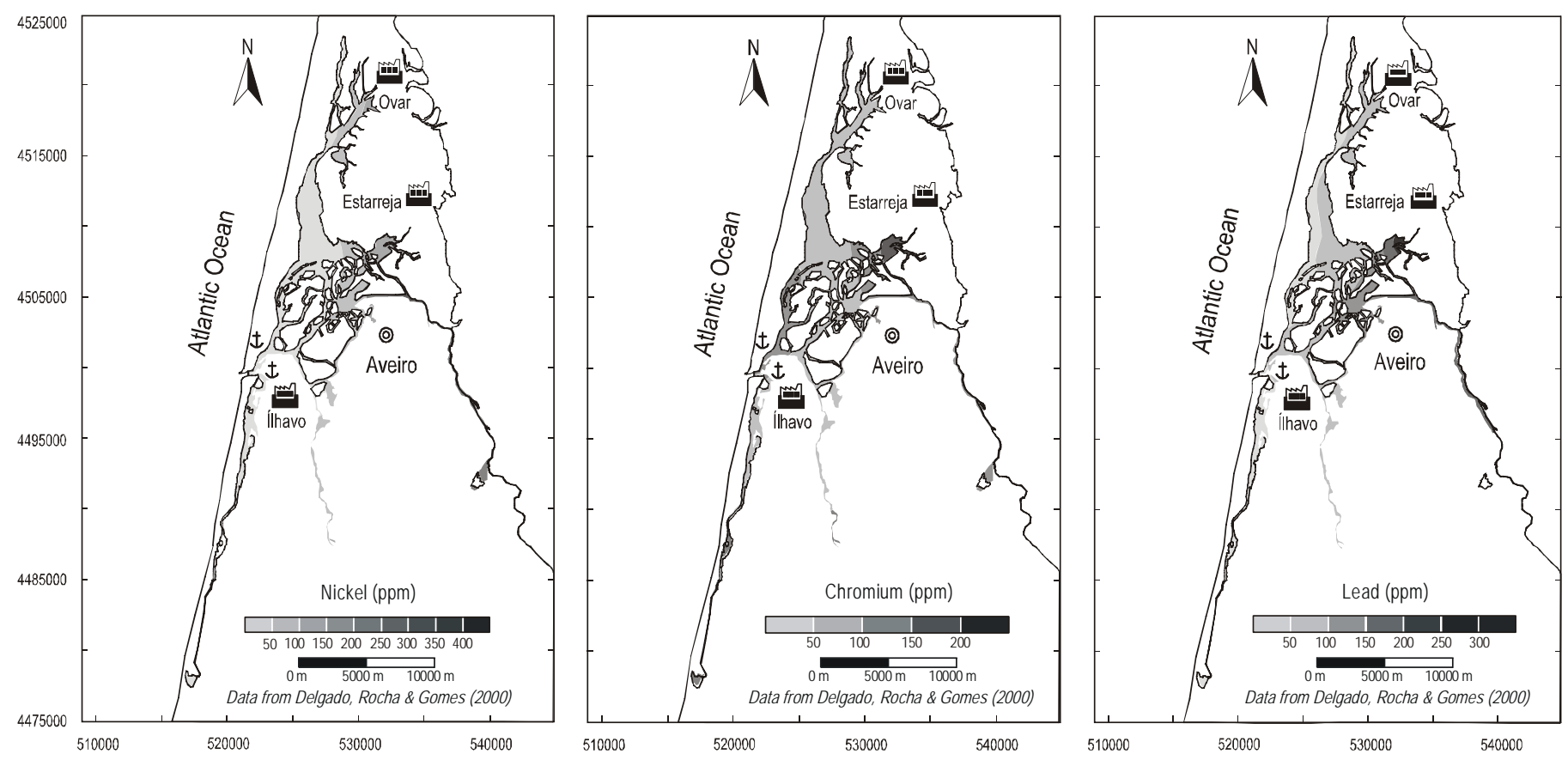

Figure 8. Dispersion maps of $\mathrm{Ni}, \mathrm{Cr}$ and $\mathrm{Pb}$; data from Delgado et al. (2000).

Figura 8. Mapas de dispersión de Ni, Cr y Pb; datos de Delgado et al. (2000).

Regarding the relationship between trace metal concentrations and clay mineral distribution, it was found that higher contents of trace metals do occur in zones where the sediments are richer in clay minerals, displaying higher specific surface areas and cation exchange capacities, in relation to the una tendencia ligera a dispersarse del área de Estarreja y a confinarse en la zona central de la laguna.

En conclusión, la distribución bien localizada de los elementos vestigiales analizados sugiere que la actividad antropogénica significativa (particularmente industrial) es el 
existence of smectite, illite-smectite, vermiculite, illitevermiculite interstratifications and vermiculite-Al hydroxide interlayers (Delgado et al., 1992, 1996; Gomes and Delgado, 1993; Rocha et al., 2000).

Mapping the sediment contents of $\mathrm{Cu}, \mathrm{Ni}, \mathrm{Pb}, \mathrm{Zn}$ and $\mathrm{Cr}$ from the Aveiro Lagoon channels and comparing with those obtained in the 1980s by Delgado et al. (2000) (figs. 7, 8), we can conclude that $\mathrm{Cu}$ and $\mathrm{Zn}$ exhibit a tendency to disperse from the Ovar and Estarreja source areas and simultaneously to be more confined, with high contents in the north sector (near Ovar); Ni shows a dispersion, mainly from the Estarreja source area and from the Lower Vouga basin; Pb exhibits a slight tendency to disperse from the Estarreja source area and to a confinement in the central zone of the lagoon.

In summary, the well-located distribution of the trace metals analyzed points to a relevant anthropogenic activity (particularly industrial) as the main factor responsible for the geochemical signature observed in the surface sediments from Aveiro Lagoon, in regards to trace metals; however, the contents of the referred metals in the same sediments also denote some hydrodynamic control due to the silting up of the lagoon channels. Evidence was found that heavy metal concentrations are higher in the more confined (therefore less hydrodynamic) channels, where the bottom sediments are richer in finer particles (silty and clayey) and display a mineralogical composition characterized by high contents of clay minerals, displaying higher specific surface areas and cation exchange capacities (such as smectite, illite-smectite, vermiculite, illite-vermiculite interstratifications and vermiculite-Al hydroxide intergrades). There is some preferential concentration of heavy metals in sediments richer in the finest fractions; therefore, higher clay contents favour heavy metal concentrations.

\section{Acknowledgements}

The present work was supported by project PRAXIS 2/2.1/ MAR/1750/95 ("Estudo integrado da transferência e efeitos de contaminantes em sedimentos, Ria de Aveiro”).

\section{References}

Azevedo, M.C.C. (1999). Contribuição para a Caracterização Ambiental da Área Envolvente do Complexo Químico de Estarreja. M.Sc. thesis, University of Aveiro, Aveiro, Portugal.

Barahona, E. (1974). Arcillas de ladrillería de la provincia de Granada: Evaluación de algunos ensayos de materias primas. Ph.D. thesis, Granada University, Spain, 398 pp.

Barradas, J.J.M. (1992). Geoquímica de elementos maiores e vestigiais em amostras de solos, sedimentos de Vale e águas subterrâneas. Contribuição para a Caracterização Ambiental da Zona Envolvente ao Complexo de Estarreja. Ph.D. Thesis, University of Aveiro, Aveiro, 204 pp.

Batista, A.C., Ferreira da Silva, E.A., Azevedo, M.C.C., Sousa, A.J. and Cardoso-Fonseca, E. (2002). Soil data analysis from central Portugal by principal component analysis and geostatistical techniques. Geochemistry: Exploration, Environment, Analysis, 2: 15-25. principal factor que determina la huella geoquímica observada en los sedimentos superficiales de la Laguna de Aveiro, en cuanto a los elementos vestigiales; sin embargo, los contenidos de éstos en los mismos sedimentos indica también algún control hidrodinámico debido al enterramiento de los canales de la laguna. Se encontró evidencia de que las concentraciones de metales pesados son mayores en los canales más confinados (y por tanto menos hidrodinámicos), donde los sedimentos del fondo son más ricos en partículas finas (limo y arcilla) y muestran una composición mineralógica caracterizada por altos contenidos de minerales arcillosos, con mayores áreas superficiales específicas y capacidades de intercambio catiónico (como interestratificaciones de esmectita, ilitaesmectita, vermiculita, ilita-vermiculita, e intercapas de vermiculita e hidróxido de $\mathrm{Al}$ ). Existe cierta concentración preferencial de metales pesados en los sedimentos más ricos en fracciones finas; por tanto, mayores contenidos de arcilla favorecen las concentraciones de metales pesados.

\section{Agradecimientos}

El presente trabajo recibió apoyo del proyecto PRAXIS 2/ 2.1/MAR/1750/95 ("Estudo integrado da transferência e efeitos de contaminantes em sedimentos, Ría de Aveiro”).

Traducido al español por Christine Harris.

Corrochano, A., Galera, M., Bernardes, C.A. y Barba, P. (1997). Características de la llanura intermareal asociada al canal de Mira (dominio interno del complejo de isla-barrera de Aveiro, Portugal). Geogaceta, 22: 47-50.

Corrochano, A., Galera, M., Jaen, P., Barba, P. y Bernardes, C.A. (1999). Facies y evolución holocena reciente en el canal mareal de Mira (Portugal). Rev. Soc. Geol. España, 12(1): 123-135.

Costa, C. and Jesus, C. (1999). Case study of heavy-metals pollution in Estarreja (Portugal). International Conference on Investigation Methods on Soil Contamination, Lisbon, 10.

Davis, J.C. (1986). Statistics and Data Analysis in Geology. Wiley, New York, 646 pp.

Delgado, H., Rocha, F. and Gomes, C. (1992). Evolution of the Aveiro Lagoon during the last 500 years based on clay mineralogy. Min. Petr. Acta, Vol. XXXV-A: 105-110.

Delgado, H.. Rocha, F. and Gomes, C. (1994). New data on heavy metals in the sediments of the Aveiro Lagoon. Proc. Littoral 94 (Eurocoast, Lisbon), pp. 516-518.

Delgado, H., Rocha, F. and Gomes, C. (1996). Statistical data analysis relating heavy metal concentrations and distribution to recent sediment nature and composition in the Aveiro Lagoon (Portugal). Proc. IVth Intern. Symp. on the Geochemistry of the Earth's Surface, Int. Assoc. Geochem. Cosmochem./Univ. Leeds, pp. 383-388.

Delgado, H., Rocha, F. and Gomes, C. (2000). Modelos de dispersão de metais pesados nos sedimentos recentes da laguna de Aveiro e sua relação com a composição e textura dos sedimentos. In: A. Duarte, C. Vale and R. Prego (eds.), Estudos de Biogeoquímica na Zona Costeira Ibérica (Actas IX Sem. Ibérico Quím. Marinha), Univ. Aveiro, pp. 325-336. 
Ferreira, M.M.I. (1993). Mercúrio em Solos na Área do Complexo Químico de Estarreja. M.Sc. thesis, University of Aveiro, Aveiro, Portugal.

Ferreira da Silva, E.A. (1989). Impacte Ambiental de Elementos Maiores e Vestigiais no Aquífero Superficial de Estarreja: Caracterização da Zona Envolvente ao Complexo de Estarreja. Provas de Aptidão Científica e Capacidade Pedagógica. Departamento de Geociências, Universidade de Aveiro, Portugal.

Garrett, R.G. (1973). The determination of sampling and analytical errors in exploration geochemistry. Econ. Geol., 68: 282-283.

Gomes, C. S. F. and Delgado, H. M. S. (1993). Heavy metals in the sediments of the Aveiro lagoon (Portugal): sources and relationships with clay minerals. Chem. Geol., 107(3-4): 423-426

Imbrie, J. and Van Andel, T.H. (1964). Vector analysis of heavymineral data. Bull. Geol. Soc. Am., 75: 1131-1156.

Jöreskob, K.G., Klovan, J.E. and Reyment, R.A. (1976). Geological Factor Analysis. Elsevier, Amsterdam, 178 pp.

Lecomte, P. and Sondag, F. (1980). Regional geochemical reconnaissance in the Belgian Ardennes, secondary dispersion patterns in the stream sediments. Miner. Deposita, 15(1): 47-60.

Mellinger, R.M. (1979). Quantitative X-ray diffraction analysis of clay minerals. An evaluation. Saskatchewan Research Council, Canada, SRC Rep. G-79: 1-46

Mezzadri, G. and Saccani, E. (1989). Heavy mineral distribution in Late Quaternary sediments of the southern Aegean sea: Implications for provenance and sediment dispersal in sedimentary basins at active margins. J. Sediment. Petrol., 59: 412-422.
Pevear, D.R. and Mumpton, F.A. (1989). Quantitative mineral analysis of clays. CMS Workshop Lectures, 1 . The Clay Minerals Soc., Colorado (USA).

Reyment, R. and Jöreskob, K.G. (1993). Applied Factor Analysis in the Natural Sciences. Cambridge Univ. Press, Cambridge, 369 pp.

Rocha, F. and Gomes, C. (1991). Clay mineralogy used for the definition of paleosurfaces in the Tertiary and Quaternary of the Aveiro sedimentary basin. Mémoires des Sciences de la Terre, Ecole des Mines de Paris, 18: 31-38.

Rocha, F. and Gomes, C. (1992). Fibrous clay minerals as morphoclimatic markers in the Aveiro sedimentary basin. Min. Petr. Acta, Vol. XXXV-A: 217-226.

Rocha, F. and Gomes, C. (1994). Discrimination of Tertiary and Quaternary lithostratigraphical units in the Aveiro region on the basis of clay minerals. Acta Univ. Carolinae Geol. (Prague), 38: 381-390.

Rocha, F., Galhano, A.C. and Gomes, C. (1999). Characterization and datation of Holocene mud layers from the urban area of Aveiro (Portugal). Cuatern. Geomorfol., 13(1/2): 45-52.

Rocha, F., Bernardes, C. and Delgado, H. (2000). Caracterização textural e mineralógica dos sedimentos da laguna de Aveiro, Portugal. Abstr. " $3^{\circ}$ Simp. Margem Contin. Atlant. Ibérica” (Univ. Algarve, Faro): 17-18.

Schultz, L.G. (1964). Quantitative interpretation of mineralogical composition from X-ray and chemical data for the Pierre Shale. US Geol. Surv. Prof. Paper, 391-C: 1-31.

Thorez, J. (1976). Practical identification of clay minerals. Ed. G. Lelotte, Belgique. 